\title{
Referral Incentives in Crowdfunding
}

\author{
Victor Naroditskiy Sebastian Stein Mirco Tonin \\ Long Tran-Thanh Michael Vlassopoulos Nicholas R Jennings
}

\author{
University of Southampton
}

\begin{abstract}
Word-of-mouth, referral, or viral marketing is a highly sought-after way of advertising. In this paper, we investigate whether such marketing can be encouraged through incentive mechanisms, thus allowing an organisation to effectively crowdsource their marketing. Specifically, we undertake a field experiment that compares several mechanisms for incentivising social media shares in support of a charitable cause. Our experiment takes place on a website promoting a fundraising drive by a large cancer research charity. Site visitors who sign up to support the cause are asked to spread the word about it on Facebook, Twitter or other channels. They are randomly assigned to one of four treatments that differ in the way social sharing activities are incentivised. Under the control treatment, no extra incentive is provided. Under two of the other mechanisms, the sharers are offered a fixed number of points that help take the campaign further. We compare low and high levels of such incentives for direct referrals. In the final treatment, we adopt a multi-level incentive mechanism that rewards direct as well as indirect referrals (where referred contacts refer others). We find that providing a high level of incentives results in a statistically significant increase in sharing behaviour and resulting signups. Our data does not indicate a statistically significant increase for the low and multi-level incentive mechanisms.
\end{abstract}

\section{Introduction}

Within just a few years, we have seen a tremendous rise of online social networks, which have made sharing information easy and natural. A message that is shared on social networks can reach a large audience without the help of traditional media outlets. Furthermore, the message may be targeted towards the right audience, as people sharing it are likely to have friends with similar interests (McPherson, Smith-Lovin, and Cook 2001). A recent social experiment, the DARPA Network Challenge (Defense Advanced Research Projects Agency 2010), provided an example of how powerful this method of sharing information can be: a seemingly impossible task of locating 10 red weather balloons

Copyright (C) 2014, Association for the Advancement of Artificial Intelligence (www.aaai.org). All rights reserved. placed at secret locations throughout the US was solved within 9 hours.

Reaching audiences through social media is particularly important for organisations that do not have a large marketing budget. For example, many charitable drives, crowdfunding or crowdsourcing projects (Douceur and Moscibroda 2007) rely on existing participants to recruit new donors or members. The Guardian ${ }^{1}$ reports that $22 \%$ of all donations on a popular charitable giving platform, JustGiving, originate from posts current donors make on Facebook.

In this work, we ask the question of how to effectively crowdsource marketing in this way, i.e., how to incentivise the sharing of a particular message on social media. To this end, we undertake a natural field experiment where we test the performance of three incentive mechanisms. The compared mechanisms encourage existing users of a website to post a message on Facebook, Twitter or other channels, in order to attract new users. The mechanisms offer a certain reward for each new signup resulting from a user's social media post. This reward is expressed in terms of points and differs between the various mechanisms as follows:

- 1 extra point for each contact who signs up (low treatment),

- 3 extra points for each contact who signs up (high treatment),

- 1 extra points for each contact who signs up, 1/2 for each contact of a contact, 1/4 for each contact of a contact of a contact and so on (recursive treatment),

- and a base case treatment providing no additional incentive (control treatment).

The experiment is run in the domain of charitable crowdfunding, where the goal of sharing a message is to generate support for a charitable cause described on a website that we designed. The users that arrive at the website are asked to sign up to "donate a click". Each click generates 1 point that together with the points generated by our referral mechanisms are used to measure the success of the fundraising campaign. Once a threshold of 1,000 points is reached, $£ 500$ is donated to support the drive. Once the next target of 10,000 points is reached, another $£ 500$ is donated.

\footnotetext{
${ }^{1}$ http://gu.com/p/38mpx
} 
We include both the 1-point and the 3-point mechanisms to test whether the exact amount of points matters for the referral activity. The recursive or multi-level incentive mechanism offers stronger incentives than the 1-point mechanism (as it offers the same number of points for direct referrals, but also rewards indirect referrals). However, how it compares to the 3-point mechanism depends on a user's social network. Indeed, either the recursive or the 3-point mechanism may generate more points for the referrer. The recursive mechanism is included to see if multi-level incentives appeal to users. Furthermore, the recursive mechanism recently generated a lot of attention as the winning strategy of the DARPA Network Challenge (Pickard et al. 2011).

We evaluate mechanisms along three dimensions. First, we look at the effectiveness of each treatment at encouraging participants to make referrals, as measured by the number of treatment participants who clicked one of the share buttons our website provides. The second dimension is the number of referred visitors to the page, and the third one is the number of those visitors who signed up.

Our main findings are that incentives make a difference in this context, and the exact amount or magnitude of the incentive is important. The 3-point incentive mechanism outperformed the control treatment on all of the metrics we considered. The recursive incentive mechanism was not any more successful than a simple 1-point mechanism. One might expect that the presence of any kind of incentives to share should be enough for most people, while further differentiation into 1,3 , or recursive points should have little bearing. However, our results suggest that offering 3 points results in more engagement than offering 1 point or compensating according to the recursive mechanism.

The paper is organised as follows. First, we cover related work. Then, we give details of our experimental design and the execution of our experiment. Finally, we present our results, followed by a discussion of our findings.

\section{Related Work}

There is little existing empirical work on comparing incentives for referrals on social media. In a recent study, Castillo, Petrie, and Wardell (2014) also conduct a field experiment in the charitable giving context. The authors describe a field experiment that compared mechanisms offering to donate a fixed amount if a user posts about the cause on Facebook. The authors find that offering $\$ 1$ for posting on Facebook encourages the action. However, a cost-benefit analysis reveals that under this referral program, each extra dollar donated by referred users costs more than $\$ 1$ in donations by the company to incentivise referrals. Unlike our study, the referral compensation money is donated based on the posting action, and not based on the impact it generates through donations from referred users. Also, our point-based compensation does not directly map to money. The points collected only result in a donation if a threshold is reached.

The question of referral schemes received a lot of interest in the theoretical community. Kleinberg and Raghavan (2005) study a model where incentives must be provided for users to propagate a question until a node that knows the answer is reached, and Cebrian et al. (2012) consider the use of recursive mechanisms in this context. Naroditskiy et al. (2012) provide a theoretical justification for the recursive mechanism used in the DARPA Network Challenge, and desirable properties of a referral scheme have been posed in (Douceur and Moscibroda 2007). The question of sybilproofness of incentive schemes has been considered in a series of papers (Babaioff et al. 2012; Drucker and Fleischer 2012; Chen et al. 2013; Lv and Moscibroda 2013). However, these papers concentrate on theoretical issues and do not investigate or compare referral mechanisms empirically with real users.

Goel, Watts, and Goldstein (2012) analyze diffusion patterns in seven online domains for the degree and depth of propagation. They find that viral reach accounts for a small fraction of users compared to the users reached directly through media or marketing. Furthermore, whatever viral reach occurs is mostly limited to users just one referral away from the users reached directly through media. In other words, referrals through referred audiences are extremely rare. Our findings confirm those results: our referred users did not generate any successful referrals. While viral reach is dreamed for by many, a more realistic goal is an increased reach through one-step referrals. Our research is helping choose the right incentives for this.

This paper contributes to the economics literature on charitable giving and fundraising, summarized in List (2011). This literature has used field experiments to analyze the effectiveness of various mechanisms used in fundraising, ranging from incentives schemes like matching grants (Karlan and List 2007) to social pressure (DellaVigna, List, and Malmendier 2012) and social information (Shang and Croson 2009). In a recent paper, Lacetera, Macis, and Mele (2014) look specifically at online fundraising and show that the effect of broadcasting donors' activities on online networks is due to homophily rather than social contagion effects. Evidence that personal referrals are important in the fundraising context is presented by Meer (2011). More generally, a number of studies have shown that peer pressure has an impact on charitable giving (Frey and Meier 2004; Shang and Croson 2005; Smith, Windmeijer, and Wright 2014).

There is also a connection to the literature on threshold public goods, i.e., public goods that are provided only if a stated threshold level of contributions is reached. In our setting, there is a threshold in terms of points to release a charitable donation and we vary how referrals contribute to reaching the threshold. Related to this point, Cadsby and Maynes (1999) find that a high threshold discourages provision, while high rewards significantly increase contributions and provision, while Croson and Marks (2000) develop the concept of step return, defined as the ratio of an individual's value of the public good to his share of the cost, and find that a higher step return leads to more contributions.

In a business context, Albuquerque et al. (2012) observe that referral activities of publishers on an online magazine platform bring significant revenue to the platform. One of the recommendations of the paper is to stimulate referral activity with incentive schemes. Schmitt, Skiera, and Van den Bulte (2011) find that referred customers are more valuable 
to a bank than non-referred customers. A reason for this is the targeting ability of crowdsourced referrals. Since an existing customer has information about the bank and about his friends, he only refers friends whose needs match what the bank provides. Domingos and Richardson (2001) provide a framework for estimating the value of each customer, taking into account the social influence that customer has on his social network.

Our work also complements the growing literature on crowdfunding, the solicitation of money or resources through social media to support a particular project (Gerber et al. 2014; Belleflamme, Lambert, and Schwienbacher 2013). Researchers typically focus on how to efficiently motivate the set of financial supporters (i.e., funders), who are already in touch with the crowdfunding project, to increase their support. In particular, Agrawal, Catalini, and Goldfarb (2011) investigate how one should take into consideration the geographical distance between the project owners and the funders. Mitra and Gilbert (2014) study the language and the phrases used in the description of the project, and their impact on the success of the corresponding crowdfunding project. Belleflamme, Lambert, and Schwienbacher (2013) look at the compensations a project owner should provide for funders (e.g., fixed, pre-ordered products, or profit share), in order to increase the total amount of monetary pledges. In a similar way, Ordanini et al. (2014) investigate how project owners should interact with the crowd to improve the efficiency of the project.

On the other hand, referrals have the potential to significantly expand not just the set of funders, but also raise the general awareness of a particular project, both of which have been identified as key motivators for project owners to participate in crowdfunding (Gerber and Hui 2013). In related work, the social capital of a project owner, measured by the number of their Facebook contacts, is shown to be significantly correlated with the success of a project (Giudici, Guerini, and Rossi Lamastra 2013; Mollick 2014). Through interviews, Hui, Gerber, and Gergle (2014) confirm that the efficient exploitation of a project owner's social network is often instrumental to the success of a crowdfunding project and highlight the importance of obtaining endorsement by contacts that are influential within their own network. In this paper, we build on these observations and investigate how to effectively encourage such referrals, thereby reaching beyond the project owner's direct contacts.

While crowdfunding is a particular application in which project owners can benefit from using their social media contacts, there have been more general studies on how users access resources within their social networks. In a laboratory study, Jung et al. (2013) show that the specific type of social capital a user has access to matters - those who report that they have some close friends within their network receive more responses to a simple favour than those who do not. Surprisingly, the actual number of friends does not matter, but those who frequently asked questions in the past also receive more responses. The researchers also show that a user's rhetorical strategy for asking for a favour matters. This study is complementary to our work, as it examines the factors that contribute to an individual's success at eliciting a response from their social network. In contrast, we also examine how to incentivise users to post a message in the first place.

In general, posting messages on social networks can incur a social cost, as users do not want to waste the time or effort of others and because they often care about how they are perceived (Sleeper et al. 2013). Rzeszotarski and Morris (2014) measure this social cost explicitly by offering users a monetary reward for asking their followers on Twitter to answer questions. They show that a higher monetary reward leads to a higher probability of users asking these questions. However, in this work, we consider referrals rather than generic questions, and we examine non-monetary incentives.

\section{Field Experiment}

In order to compare a range of mechanisms for incentivising referrals, we partnered with Cancer Research UK (CRUK), one of the UK's largest charitable organisations, to carry out a natural field experiment. To this end, we set up a website to raise awareness of one of CRUK's existing fundraising campaigns. The website provided an indirect incentive for visitors to engage with it by adding a point to an overall total displayed on the website whenever a new user signed up. This total was then used to release donations from an outside donor to the CRUK campaign - whenever certain point thresholds were reached, a new donation was made.

After signing up, users were randomly assigned to one of a set of referral mechanisms. Here, some of the users were able to add further points to the common total by successfully referring their social contacts to the website. This allowed us to test whether such additional incentives can increase the probability of a successful referrals, and, by testing various mechanisms, whether the magnitude of the reward is significant and whether recursive schemes offer a benefit in this setting.

In the following, we describe the setup of our field experiment and the tested referral mechanisms in more detail.

\section{Website}

Our website, http://outruncancer.co.uk/, was set up in September 2013 in consultation with CRUK and provided visitors with information about oesophageal cancer and a particular CRUK fundraising campaign, "The Cancer Marathon". This campaign was initiated by six medics at the Southampton General Hospital, who were participating in the New York Marathon in November 2013 to raise money for research on oesophageal cancer.

The stated purpose of our website was to support the campaign by spreading awareness about it and thereby attract further donations from the public. To achieve this and encourage engagement with our site, visitors could contribute one point to a total amount prominently displayed on the website simply by signing up. This did not incur a financial cost for them and involved either entering an email address and password, or signing in through existing Facebook or Twitter accounts. Importantly, we pledged to make several donations of $£ 500$ each to CRUK's fundraising campaign, as soon as certain thresholds in the total number of points generated by all users were reached: 


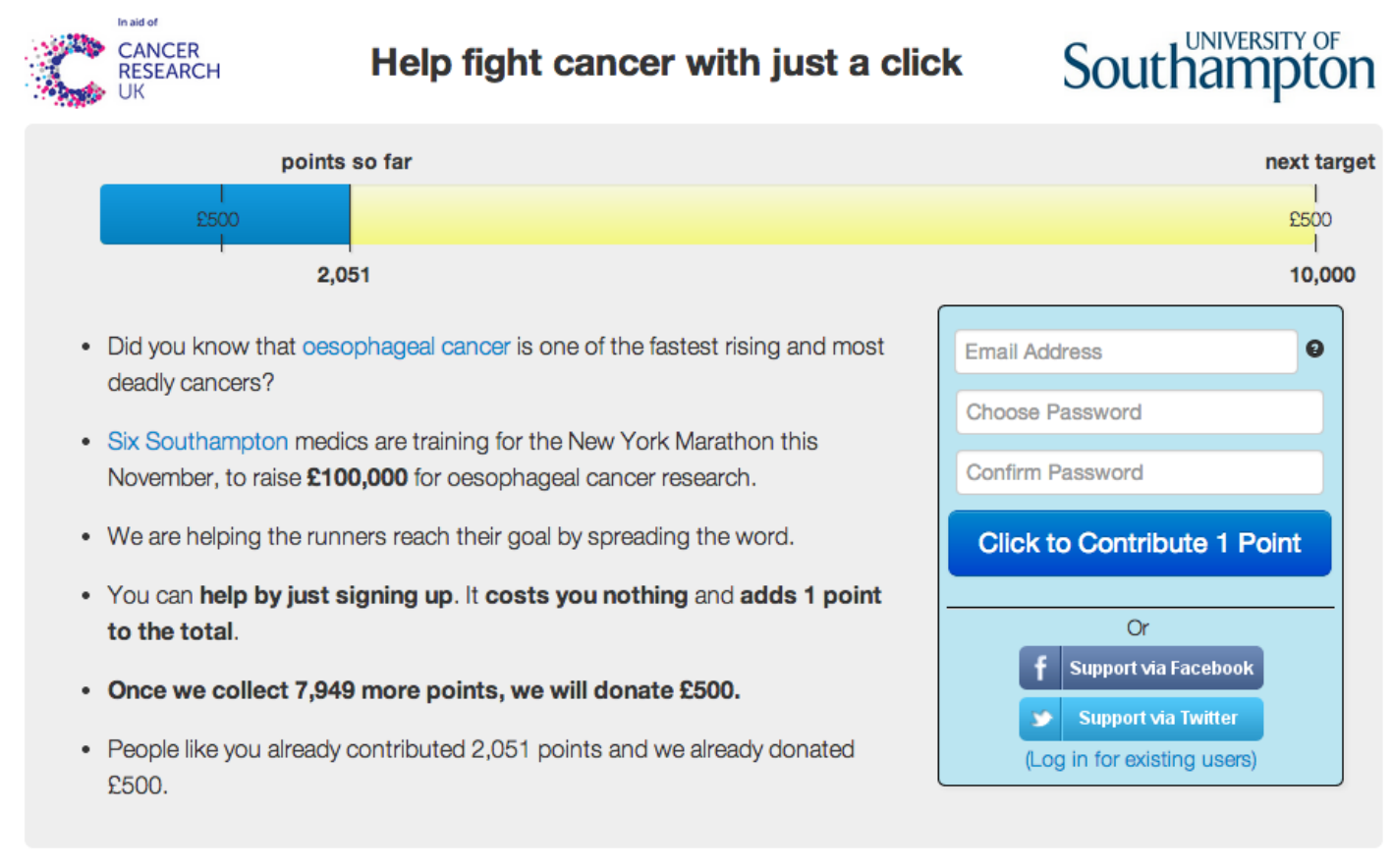

Figure 1: Main panel of the landing page.

1. $£ 500$ when 1,000 points were reached in total

2. $£ 500$ when 10,000 points were reached in total

3. $£ 500$ when 50,000 points were reached in total

4. $£ 500$ when 100,000 points were reached in total

We chose this scheme, in order to cope with varying total amounts of visitors to our site. The first threshold was relatively low, in order to be able to generate at least one donation, while the next thresholds were spaced increasingly further apart, to ensure that the experiment did not have to terminate due to running out of money for donations.

Figure 1 shows the main part of the initial landing page (the full page is shown in Figure 9 in the appendix). Here, a prominent box displayed the current progress to the next donation, a summary of how the website works and a registration panel. Below this (see Figure 9), there were expandable sections offering more details about the campaign and the website. Importantly, as the experiment was designed as a natural field experiment, the website did not disclose that (anonymised) data from users would support research on referral incentives. This was crucial, because knowledge of the research could have altered the users' referral behaviour.

Apart from the dynamically updated total number of points, all visitors to the website received exactly the same information, and there was no explicit mention of referrals. This was done to avoid any priming of the visitors, and to mitigate self-selection bias regarding referral behaviour. The only varying parameter - the total number of points - did not affect our results as we only used observations from the people who participated after the first target of 1,000 points had been reached and when the next target of 10,000 was very far away (the experiment ended with the total number of points just over 2,000).

\section{Referral Mechanisms}

Once a visitor signed up to the website, they were presented with a page thanking them for their contribution and suggesting a number of ways they can help the fundraising campaign (Figure 2 shows the main part of this page, while the full page is shown in Figure 10 in the appendix). In particular, they were asked to invite their friends via a range of channels: by copying and sending a personalised link, by sharing a link on Facebook or Twitter, or by sending an email with the link.

Here, we provided some users with additional incentives, depending on the treatment they were randomly allocated to. This random allocation took place on signup and used a simple blocked randomisation scheme, whereby the allocation mechanism repeatedly cycled through random permutations of the available treatments (thus ensuring a near-balanced allocation to treatments).

The treatments we considered were as follows:

- Control: Users on this treatment were offered no additional referral incentives.

- Low: Users on this treatment were told that 1 additional point would be added to the total when they successfully referred someone else to the website (in addition to the signup point generated by that contact).

- High: Similar to Low, but users were offered 3 additional points.

- Recursive: Users were told that $0.5^{n-1}$ additional points would be added to the total when they successfully re- 


\section{Thank you very much!}

You have contributed 1 point to fight oesophageal cancer. $£ 500$ will be donated once 7,948 more points are generated.

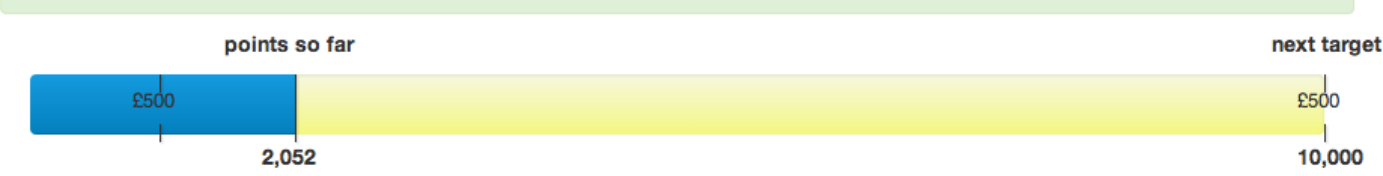

\section{Help reach the target faster}

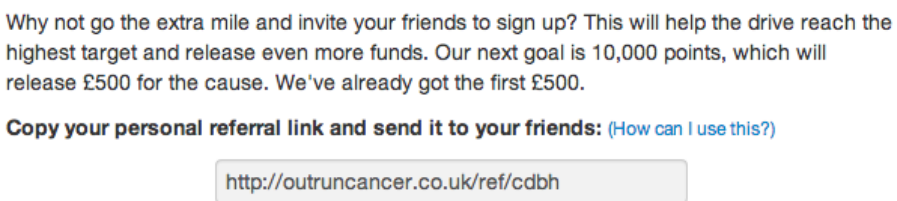

Or use the following buttons:

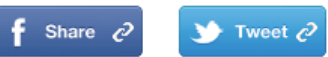

How much awareness can you generate?

- 0 of your friends signed up

Figure 2: Referral options after signing up (control treatment shown).

ferred someone else to the website, including through indirect referrals, where $n$ is the distance to the user. ${ }^{2}$ Thus, if the user successfully referred another contact, 1 additional point would be added to the total. If that referred contact successfully referred someone else, 0.5 additional points would be added, and so on.

The wordings of these treatments, as shown to the users, are summarised in Figure 3. Referrals were tracked through a unique ID that was allocated to each user and then included in the referral links generated through the four referral methods on the website (direct link, Facebook, Twitter or email). These links also encoded which of the four methods was used, allowing us to track, for each referred user, who they were referred by and through which referral method. Overall, we recorded the following three key metrics for each user:

- Number of channels used: How many of the four referral methods were initiated by the user on our website. We record this when the user clicks on one of the buttons (or the referral link) shown after signing up. ${ }^{3}$

- Number of resulting visits: How many visits were subsequently generated by people following the user's unique referral link (but did not necessarily sign up).

- Number of resulting signups: How many new users subsequently signed up through the user's referral link (this

\footnotetext{
${ }^{2}$ We say user A indirectly referred B, if B was not (directly) referred by A, but by another user C, which was either referred by $\mathrm{A}$ or is also an indirect referral of $\mathrm{A}$. The distance between $\mathrm{A}$ and $\mathrm{B}$ is the length of the resulting referral chain (a direct referral has distance 1).

${ }^{3}$ Note that this is not an exact measure of referral messages posted, as users may still cancel the message on the social media website before posting or may never share the link.
}

is number of the user's direct referrals).

Note that for all four treatments, there is an implicit incentive for users to refer contacts to the website, as all signups generate 1 point towards the total. The low, high and recursive treatments add an additional incentive on top of this. The first two of these, low and high, are simple schemes that we chose to investigate whether the magnitude of this incentive is relevant - in the first case, it is equivalent to the original incentive offered to the user for signing up; in the second case, it is significantly higher than the original incentive. We included the recursive scheme, because it has been used successfully in previous crowdsourcing applications (as described in Related Work) and because it may encourage users to specifically target contacts with a wide social reach. From a user's perspective, it dominates the low scheme, because it also awards 1 additional point for each direct referral, but may generate further points through subsequent indirect referrals. However, the relative attractiveness of recursive and high depends on the user's social network. For example, a user who has only one friend who is very well-connected, prefers the recursive scheme as he will be rewarded half a point on each signup of the wellconnected friend's friends.

Users were able to log back into the website at any time to view how many of their contacts had signed up and (except for the control treatment) how many additional points had been generated through this. We also gave users the option to donate directly to the Cancer Marathon campaign, which we matched one-to-one (up to $£ 10$ per user).

\section{Subject Recruitment}

The website went live in September 2013 and over the next two months, 1,577 unique users signed up, generating a total of 2,051 points (one point was generated on signup; the 


\section{Help reach the target faster}

Why not go the extra mile and invite your friends to sign up? This will help the drive reach the highest target and release even more funds. Our next goal is 10,000 points, which will release $£ 500$ for the cause. We've already got the first $£ 500$.

- For every friend who signs up, 1 additional point will be generated on your behalf.

\section{Help reach the target faster}

Why not go the extra mile and invite your friends to sign up? This will help the drive reach the highest target and release even more funds. Our next goal is 10,000 points, which will release $£ 500$ for the cause. We've already got the first $£ 500$.

- For every friend who signs up, 1 additional point will be generated on your behalf.

- For every friend of a friend who signs up, $1 / 2$ of an additional point will be generated on your behalf.

- For every friend of a friend of a friend who signs up, $1 / 4$ of an additional point will be generated on your behalf.

- ....and so on.

(a) low treatment

(b) recursive treatment

Figure 3: Explanatory texts for low and recursive treatments. Text for high treatment is identical to low except for the number of additional points (3).

$\begin{array}{lr}\text { Outrun Cancer } & 23 \text { September } 2013 \text { 13:35 } \\ \text { To: Outrun Cancer } & \text { Hide Details } \\ \text { Help cancer research with a click } & \end{array}$

Ever heard of oesophageal cancer?

It is one of the fastest rising and most deadly cancers. The University of Southampton and Cancer Research UK are working to find a cure. To raise funds for this research, six medics from the University and the Southampton General Hospital are running a marathon this November.

You too can help the fight against oesophageal cancer by visiting http://outruncancer.co.uk/go and donating a click. Every click counts towards releasing $£ 2,000$ from a sponsor. It costs you nothing, except a few seconds of your time.

For more information, visit http://outruncancer.co.uk/go, email us at outruncancer@soton.ac.uk or the Cancer Research UK Southampton Centre at southamptoncentre@cancer.org.uk.

Thank you for your support

The Outrun Cancer Team

Figure 4: Email to invite participants.

remaining 474 points were generated for successful referrals) and an additional $£ 418$ in user donations. As a result of meeting the first threshold, we donated $£ 500$, as well as $£ 408$ in matching donations (this is slightly lower than the total amount donated by the users due to the $£ 10$ limit per user).

Initial users were recruited from a range of sources. Some publicity was generated by CRUK and the medics running the Cancer Marathon, including through Twitter messages and a local radio interview. However, the bulk of the users were attracted through emails within the University of Southampton, sent to staff and student mailing lists. This email did not mention that the website was part of a research project and did not identify the academics involved in it (see Figure 4).

During the course of the experiment, we received feedback from users, indicating that the points mechanism was not explained clearly and succinctly enough. For this reason, we made a number of changes to the website in mid-October 2013 - primarily to highlight the current points collected to date at the top of the page and to emphasise their purpose. As the experimental conditions changed at that time, we focus only on the 412 participants recruited after the change in the remainder of this paper. These are mainly students from the Faculties of Humanities, Social Sciences, and Health Sciences and their referred contacts, as we emailed their internal mailing lists after the change (and had not previously contacted them). Changing the experiment half-way did not present any complications as far as the analysis of the results goes. Restarting midway can be viewed as starting the experiment at the midpoint as the first part was simply ignored and there was no contamination from it.

After the redesign, we contacted 17,686 University of Southampton students via email. There were 1,042 unique visits that resulted in 412 signups. In terms of the effectiveness of various social media channels, Twitter was responsible for generating $55 \%$ the referred traffic while Facebook brought $36 \%$ of referred page visits. However, the number of page visits that turned into signups is much higher for Facebook: $62 \%$ compared to Twitter's $8 \%$. The remaining visits and signups were generated by sharing a link without the use of the social media buttons.

There were too few donations to perform meaningful statistical analysis of differences across treatments, but we report them here for completeness. After the redesign, the total of $£ 40$ was donated by 6 users. Five donors were in the High treatment donating the total of $£ 30$ and one user who donated $£ 10$ was in the Low treatment.

\section{Results}

Table 1 summarises the total number of times all channels were used under each treatment, the number of resulting visits, and the number of resulting signups. We observe that the 3 -point treatment resulted in higher totals across the board. There is no other clear dominance across treatments. Somewhat of an outlier is a low ratio of recursive treatment's visits and signups. There were 103 visits but only 2 signups resulting in a $2 \%$ signup ratio. The corresponding ratios for the control, low, and high treatment are $14 \%, 6 \%$, and $11 \%$ respectively. Given the limited number of observations, we could not test for whether there is an association between ratio and treatment.

We present details of visits and signups in Figures 5 and 6. The histogram in Figure 6 shows how many referrers signed up exactly 1 friend, 2 friends, .., 8 friends. For the high 


\begin{tabular}{llllll}
\hline & Control & Low & High & Recursive & Total \\
\hline Channels used & 13 & 15 & 32 & 28 & 88 \\
\hline Resulting visits & 37 & 98 & 218 & 103 & 456 \\
\hline Resulting signups & 5 & 6 & 24 & 2 & 37 \\
\hline
\end{tabular}

Table 1: Total outcomes per treatment.

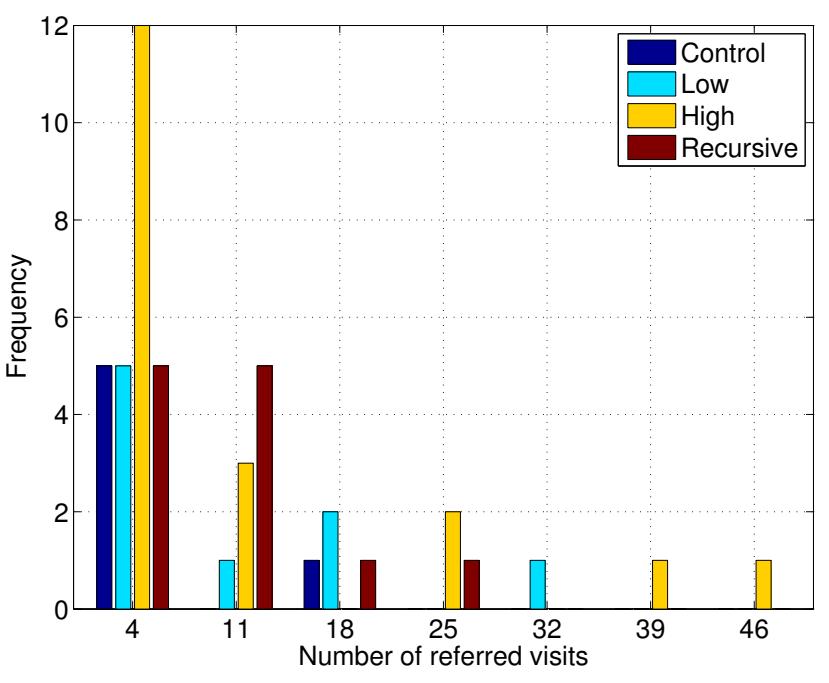

Figure 5: Histogram of visits generated per user.

treatment, there were 6 referrers who signed up 1 friend, 3 referrers who signed up 2 friends, 1 referrer who signed up 4 , and 1 referrer who signed up 8 friends. The histogram of visits in Figure 5 should be read in a similar manner: the first set of bars shows how many referrers generated between 1 and 7 referred visits, the second set of bars shows how many referrers generated between 8 and 14 referred visits, etc. For example, between 8 and 14 visits were generated by zero referrers in the control treatment, by 1 referrer in the low treatment, by 3 referrers in the high treatment, and by 5 referrers in the recursive treatment.

Next, we evaluate the results we obtained from our experiment. In particular, we focus on both the extensive and intensive margins of the behaviour under investigation. The extensive margin uses binary $(0 / 1)$ indicators that reflect whether a certain success measure is achieved. In our case, relevant indicators are whether or not a referrer generated a visit and whether or not she generated a signup. The intensive margin quantifies the degree of success by looking at the number of visits and signups generated by each referrer.

\section{Extensive Margin}

In this section we examine whether there are differences in the outcomes of interest in the extensive margin. We focus on three binary outcomes: (i) whether a referral channel was taken; (ii) whether at least one visit was generated; (iii) whether at least one signup was generated. All results regarding these three outcomes are summarised in Table 2.

With regards to referral channels, we see in the first sec-

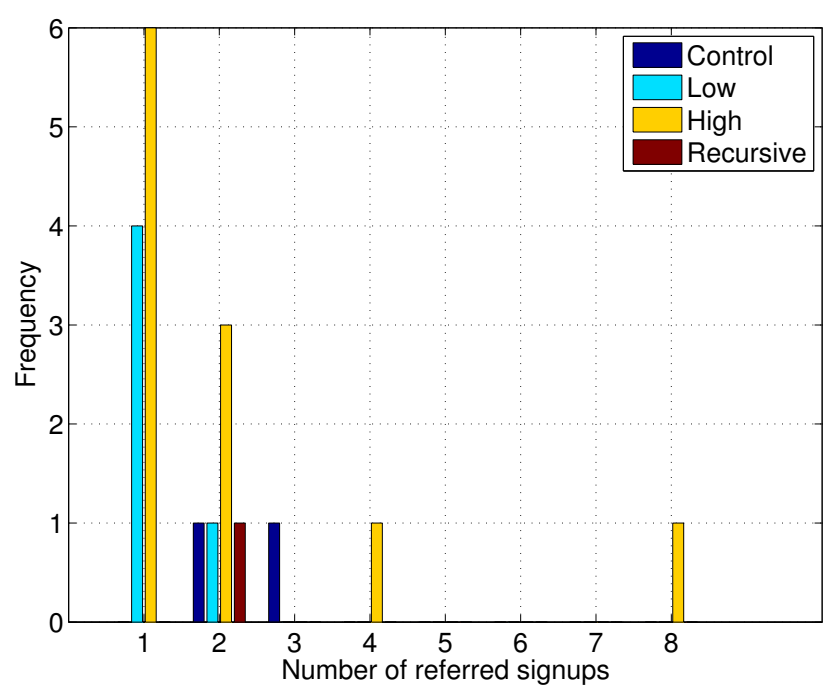

Figure 6: Histogram of signups per user.

tion of Table 2 that people in our sample used some of the tools we provided to refer other potential participants in $16 \%$ of the cases (65 of the total 412). With no incentives, this happened in only $10 \%$ of the cases, while the percentage is always higher when referral incentives are present. In particular, the high incentive treatment is successful in eliciting a referral through some channel among $21 \%$ of the people, while for the low incentive treatment the corresponding figure is $14 \%$. The recursive treatment is in between, with a figure of $18 \%$. The p-value of a chi-squared test is, at 0.106 , which does not allow us to reject the null hypothesis of independence between the likelihood of undertaking some referral channel action and the various treatments.

Out of the $16 \%$ of people who used some of the referral tools, $75 \%$ were successful in generating some visits to the website. That is, out of 65 people who used a referral channel, 46 resulted in at least one new user visiting the website (see the second section of Table 2). The effect of incentives in generating additional traffic is even more evident, with only $6 \%$ of the people in the control group generating some visits, while the figure for the high incentive group, at $18 \%$, is three times higher. Again, the low incentives are less successful (9\%) and recursive incentives are positioned in between (12\%). Figure 7 displays the percentages along with $95 \%$ confidence intervals. A chi-squared test rejects the null hypothesis of independence between the likelihood of generating some visits and the various treatments (p-value $=0.028)$.

The percentage of people who generated additional 


\begin{tabular}{llllll}
\hline & Control & Low & High & Recursive & Total \\
\hline At Least One Channel Used? & & & & & \\
\hline Yes & $10(9.8 \%)$ & $14(13.5 \%)$ & $22(21.4 \%)$ & $19(18.4 \%)$ & $65(15.8 \%)$ \\
\hline No & $92(90.2 \%)$ & $90(86.5 \%)$ & $81(78.6 \%)$ & $84(81.6 \%)$ & $347(84.2 \%)$ \\
\hline \hline Resulting Visits? & & & & & \\
\hline Yes & $6(5.9 \%)$ & $9(8.7 \%)$ & $19(18.4 \%)$ & $12(11.7 \%)$ & $46(11.2 \%)$ \\
\hline No & $96(94.1 \%)$ & $95(91.3 \%)$ & $84(81.6 \%)$ & $91(88.3 \%)$ & $366(88.8 \%)$ \\
\hline \hline Resulting Signups? & & & & \\
\hline Yes & $2(2 \%)$ & $5(4.8 \%)$ & $11(10.7 \%)$ & $1(1 \%)$ & $19(4.6 \%)$ \\
\hline No & $100(98 \%)$ & $99(95.2 \%)$ & $92(90.3 \%)$ & $102(99 \%)$ & $393(95.4 \%)$ \\
\hline \hline & & & & & \\
\hline Total Participants & 102 & 104 & 103 & 103 & 412 \\
\hline
\end{tabular}

Table 2: Referral channels, visits and signups per treatment (extensive margin).

The p-value of a chi-squared test for independence is $0.106,0.028$ and 0.004 for channels used, visits and signups, respectively.

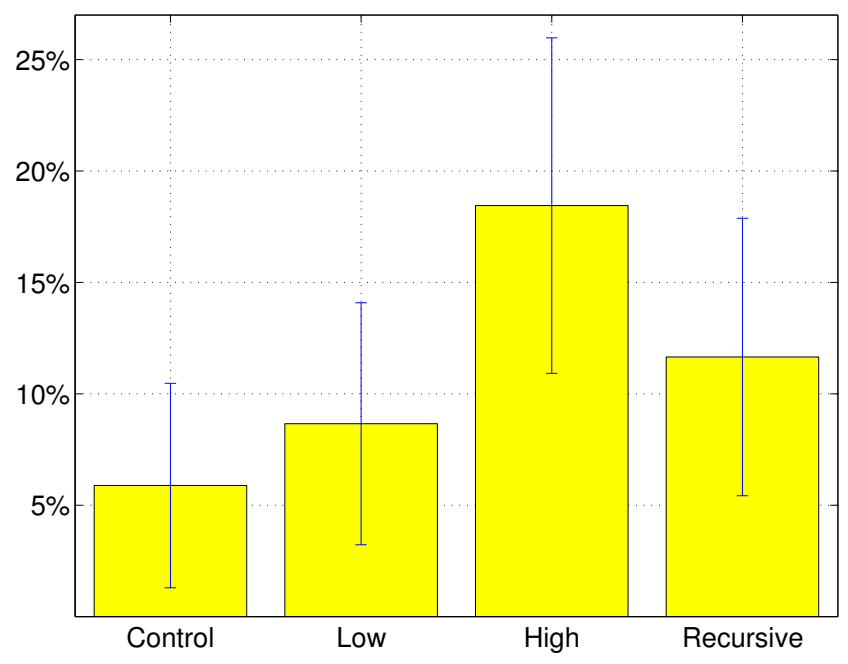

Figure 7: Percentage of participants whose referrals brought at least one website visitor. 95\% confidence intervals are shown.

signups is $4.6 \%$ (see the third section of Table 2). Again, our referral treatments seem to be effective in boosting this number. Only $2 \%$ of people assigned to the control group generated additional signups, while in the high incentives case, the figure is much higher, at $11 \%$. Low incentives are less effective (5\%), while the recursive treatment is the least effective (1\%). Figure 8 displays the percentages along with 95\% confidence intervals. Again, a chi-squared test rejects the null hypothesis of independence between the likelihood of generating additional signups and the various treatments ( $\mathrm{p}$-value $=0.004)$.

These results are confirmed by a regression analysis (Table 3), where it is evident that the high incentives treatment is effective in generating significantly more referral actions, more visits, and more signups than the control group. ${ }^{4}$ For

\footnotetext{
${ }^{4}$ We present regression analysis here and not ANOVA because it allows for pairwise comparisons between each of the treatments and the control, which is our primary interest.
}

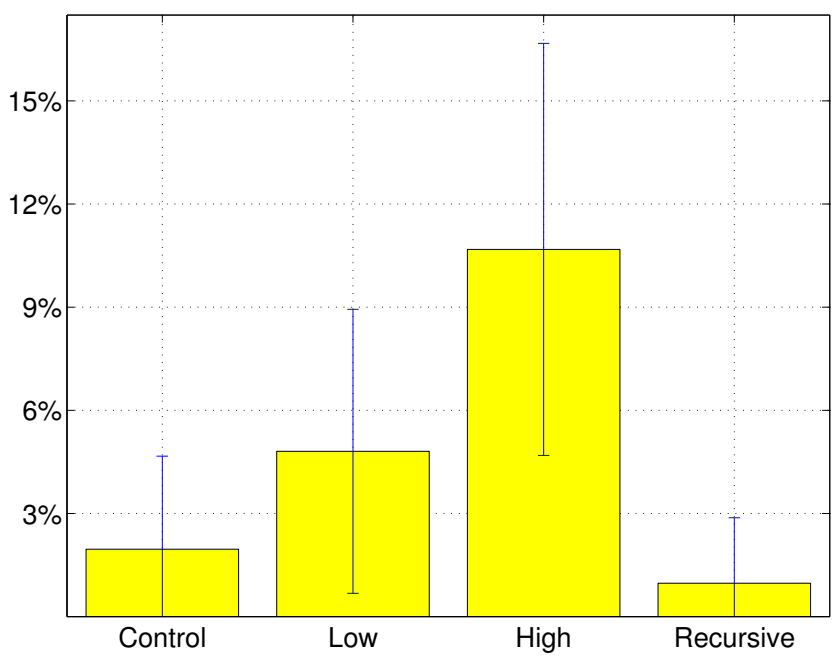

Figure 8: Percentage of participants whose referrals resulted in at least one signup. $95 \%$ confidence intervals are shown.

the other treatments, the coefficients are generally positive but not statistically significant. Note also that with regards to signups, the difference between the high incentives and the recursive treatment is also statistically significant ( $\mathrm{p}$ value $=0.007$ ), while the difference between high and low is marginally not (p-value $=0.116$ ). Finally, for visits, we find a significant difference between the coefficients of the high versus the low treatment ( $\mathrm{p}$-value $=0.04$ ).

The model we use for the analysis is a standard probit, where we fit the following equation: $\operatorname{Pr}($ signups $=1)=$ $\Phi\left(b_{0}+b_{1} *\right.$ High $+b_{2} *$ Low $+b_{3} *$ Recursive $) . \Phi$ is the cumulative normal distribution. High is a variable taking the value of 1 if observation is in High treatment, 0 otherwise. Low is a variable taking the value of 1 if observation is in Low treatment, 0 otherwise. Recursive is a variable taking the value of 1 if observation is in Recursive treatment, 0 otherwise. The omitted treatment is Control and, therefore, for that treatment we report the coefficient on the constant, $b_{0}$. The asterisks indicate the significance of tests on whether 


\begin{tabular}{lllll}
\hline & Control & Low & High & Recursive \\
\hline Referral channels & $-1.293^{* * *}(0.17)$ & $0.188(0.23)$ & $0.499^{* *}(0.22)$ & $0.394 *(0.22)$ \\
Visits & $-1.565^{* * *}(0.20)$ & $0.202(0.27)$ & $0.666^{* * *}(0.25)$ & $0.372(0.26)$ \\
Signups & $-2.062 * * *(0.29)$ & $0.398(0.36)$ & $0.818^{* *}(0.33)$ & $-0.276(0.47)$ \\
\hline
\end{tabular}

Table 3: Regression analysis of the treatments.

Probit regression — robust standard errors are given in parentheses. $* * *[* *](*)$ denote significance at $1,[5],(10) \%$ level.

the coefficients are different from 0.

\section{Intensive Margin}

In this section we examine whether there are differences in the outcomes of interest in the intensive margin. We focus again on three outcomes: (i) number of channels used; (ii) total number of visits generated; (iii) total number of signups generated (as described in the Referral Mechanisms Section).

Table 4 presents averages for the various outcomes across the four treatment groups. It is evident that the high incentives treatment achieves the highest average in all measures, and in particular, with regards to the number of signups. In fact, t-tests of differences in means of the various outcomes between participants in the high and the control group indicate statistically significant differences in all cases (pvalues are 0.008 for channels, 0.018 for visits and 0.069 for signups)

Finally, when we perform nonparametric tests (MannWhitney test) of differences in the distribution of the various outcomes between participants in the high and the control group we find statistically significant differences in all cases (p-values are 0.018 for channels, 0.005 for visits and 0.012 for signups). When comparing high to low treatment, we find significance differences for visits $(p=0.04)$, while for sign-ups the $p$-value is 0.11 . Also, the difference in the distribution of sign-ups of high and recursive treatments is significant ( $p$-value $=0.003$ ).

\section{Discussion}

Word-of-mouth, referral, or viral marketing is a highly sought-after way of advertising. Ideally, it would occur organically because people find the promoted message of high value. It is however notoriously difficult to design viral messages. In this paper we attempt not to optimise the message, but to find out how sharing of a given message can be stimulated.

Our finding that the 3-point mechanism is more effective than the 1-point mechanism points to the need to choose the level of compensation carefully. This is contrary to the intuition that it is not the exact level, but rather the presence of some form of incentives, which has the most effect. Consistent with this intuition, Castillo, Petrie, and Wardell (2014) find little difference in referral activity between a mechanism that pays $\$ 1$ and $\$ 5$ for a sharing action. The corresponding sharing rates are $37 \%$ and $39 \%$.

One might expect incentives to not matter at all in the context where points do not directly correspond to donations, but only matter if a sufficient number of points is reached. This is even more plausible if the threshold number of points does not appear to be within easy reach. This was the case in our experiment. All of the observations used in the data analysis came from the users who signed up after the first target of 1,000 points was reached, and when the second target of 10,000 points was far away. Indeed, the total number of points at the end of the experiment was just above 2,000. No user could reasonably expect that their friends would bring 8,000 points no matter which treatment they were part of. The result that users still reacted to the 3-point treatment more than to the 1-point or recursive treatments is even more interesting given this context.

Our mechanisms reward successful referrals and they are complementary to the mechanisms that reward referral $a c$ tions (i.e., simply sharing a message about the cause, regardless of whether this attracts new signups). Cost-benefit analysis of paying for referral actions results in cautionary lessons (Castillo, Petrie, and Wardell 2014). We conjecture that the mechanisms considered in our work can be more cost-effective. In particular, one could guarantee an arbitrary cost-benefit ratio by offering the reward as a fraction of the actual donation made by the referred donors.

From an organisation's point of view, encouraging social media shares is crowdsourcing marketing activities. Taking a broad view on this line of research, we are after learning how to encourage existing participants or customers to do marketing for the project or business. In future work, we plan to investigate this question of how to balance the benefits of an incentive scheme with its related costs. We will also explore whether gamification mechanisms, including leaderboards or badges, are effective in this context, and we will carry out further experiments in other domains, such as crowdsourcing projects and commercial websites.

As in any empirical research, and in particular in an experimental setting, there is a concern about generalizability. Do our findings apply beyond the charitable context? Would we find similar results with a different population or are university students somehow special, for instance because their social network has specific characteristics? The fact that we use a control group implies that differences between students and non-students related, for instance, to the average number of friends or the likelihood to use social networks do not matter. Nor do differences, to make another example, in the general propensity to respond to a charitable versus a non charitable cause. What our results show is a differential response to the various incentive mechanisms we test and to assess the robustness of this finding in different contexts and with different populations we are currently conducting further studies. 


\begin{tabular}{llllll}
\hline & Control & Low & High & Recursive & Total \\
\hline Action channels used & 0.1 & 0.14 & 0.26 & 0.23 & 0.18 \\
\hline Resulting visits & 0.36 & 0.94 & 2.12 & 1 & 1.11 \\
\hline Resulting signups & 0.05 & 0.06 & 0.23 & 0.05 & 0.09 \\
\hline
\end{tabular}

Table 4: Average outcomes per treatment.

\section{Acknowledgements}

We would like to thank the team at CRUK and, in particular, Hannah Fox and Tim Underwood. This work was funded by the Strategic Interdisciplinary Research Development Fund of the Faculty of Social and Human Sciences at the University of Southampton, and by the EPSRC ORCHID programme grant. This work is also supported by the British Academy Small Research Grant SG131130.

\section{References}

Agrawal, A. K.; Catalini, C.; and Goldfarb, A. 2011. The geography of crowdfunding. National Bureau of Economic Research.

Albuquerque, P.; Pavlidis, P.; Chatow, U.; Chen, K.-Y.; and Jamal, Z. 2012. Evaluating promotional activities in an online two-sided market of user-generated content. Marketing Science 31(3):406-432.

Babaioff, M.; Dobzinski, S.; Oren, S.; and Zohar, A. 2012. On bitcoin and red balloons. In Proceedings of the 13th ACM Conference on Electronic Commerce, 56-73. ACM.

Belleflamme, P.; Lambert, T.; and Schwienbacher, A. 2013. Crowdfunding: Tapping the right crowd. Journal of Business Venturing.

Cadsby, C. B., and Maynes, E. 1999. Voluntary provision of threshold public goods with continuous contributions: experimental evidence. Journal of Public Economics 71(1):53-73.

Castillo, M.; Petrie, R.; and Wardell, C. 2014. Fundraising through online social networks: A field experiment on peerto-peer solicitation. Journal of Public Economics 114(0):29 -35 .

Cebrian, M.; Coviello, L.; Vattani, A.; and Voulgaris, P. 2012. Finding red balloons with "split" contracts: robustness to individuals' selfishness. In ACM Symposium on Theory of Computing (STOC).

Chen, W.; Wang, Y.; Yu, D.; and Zhang, L. 2013. Sybilproof mechanisms in query incentive networks. In Proceedings of the fourteenth ACM conference on Electronic commerce, 197-214. ACM.

Croson, R. T., and Marks, M. B. 2000. Step returns in threshold public goods: A meta-and experimental analysis. Experimental Economics 2(3):239-259.

Defense Advanced Research Projects Agency. 2010. DARPA Network Challenge Project Report.

DellaVigna, S.; List, J. A.; and Malmendier, U. 2012. Testing for altruism and social pressure in charitable giving*. The Quarterly Journal of Economics.
Domingos, P., and Richardson, M. 2001. Mining the network value of customers. In Proceedings of the Seventh ACM SIGKDD International Conference on Knowledge Discovery and Data Mining, KDD '01, 57-66. New York, NY, USA: ACM.

Douceur, J. R., and Moscibroda, T. 2007. Lottery trees: motivational deployment of networked systems. In Murai, J., and Cho, K., eds., SIGCOMM, 121-132. ACM.

Drucker, F. A., and Fleischer, L. K. 2012. Simpler sybilproof mechanisms for multi-level marketing. In Proceedings of the 13th ACM conference on Electronic commerce, 441458. ACM.

Frey, B. S., and Meier, S. 2004. Social comparisons and prosocial behavior: Testing" conditional cooperation" in a field experiment. The American Economic Review 94(5):17171722.

Gerber, E. M., and Hui, J. 2013. Crowdfunding: Motivations and deterrents for participation. ACM Transactions on Computer-Human Interaction (TOCHI) 20(6):34.

Gerber, E. M.; Muller, M.; Wash, R.; Irani, L. C.; Williams, A.; and Churchill, E. F. 2014. Crowdfunding: an emerging field of research. In 32nd Annual ACM Conference on Human Factors in Computing Systems (CHI 2014), 1093-1098. ACM.

Giudici, G.; Guerini, M.; and Rossi Lamastra, C. 2013. Why crowdfunding projects can succeed: The role of proponents individual and territorial social capital. Available at SSRN 2255944.

Goel, S.; Watts, D. J.; and Goldstein, D. G. 2012. The structure of online diffusion networks. In Proceedings of the 13th ACM Conference on Electronic Commerce, EC '12, 623638. New York, NY, USA: ACM.

Hui, J.; Gerber, E.; and Gergle, D. 2014. Understanding and leveraging social networks for crowdfunding: implications for support tools. In CHI'14 Extended Abstracts on Human Factors in Computing Systems, 2083-2088. ACM.

Jung, Y.; Gray, R.; Lampe, C.; and Ellison, N. 2013. Favors from facebook friends: unpacking dimensions of social capital. In Proceedings of the SIGCHI Conference on Human Factors in Computing Systems, 11-20. ACM.

Karlan, D., and List, J. A. 2007. Does price matter in charitable giving? evidence from a large-scale natural field experiment. The American economic review 97(5):1774-1793.

Kleinberg, J., and Raghavan, P. 2005. Query incentive networks. In Foundations of Computer Science, 2005. FOCS 2005. 46th Annual IEEE Symposium on, 132-141. IEEE.

Lacetera, N.; Macis, M.; and Mele, A. 2014. Viral altruism? generosity and social contagion in online networks. 
List, J. A. 2011. The market for charitable giving. The Journal of Economic Perspectives 157-180.

Lv, Y., and Moscibroda, T. 2013. Fair and resilient incentive tree mechanisms. In Proceedings of the 2013 ACM symposium on Principles of distributed computing, 230-239. ACM.

McPherson, M.; Smith-Lovin, L.; and Cook, J. M. 2001. Birds of a feather: Homophily in social networks. Annual Review of Sociology 27:415-444.

Meer, J. 2011. Brother, can you spare a dime? peer pressure in charitable solicitation. Journal of Public Economics 95(78):926 - 941 .

Mitra, T., and Gilbert, E. 2014. The language that gets people to give: Phrases that predict success on kickstarter. CSCW.

Mollick, E. 2014. The dynamics of crowdfunding: An exploratory study. Journal of Business Venturing 29(1):1-16.

Naroditskiy, V.; Rahwan, I.; Cebrian, M.; and Jennings, N. R. 2012. Verification in referral-based crowdsourcing. PLOS ONE 7(10).

Ordanini, A.; Miceli, L.; Pizzetti, M.; and Parasuraman, A. 2014. Crowd-funding: transforming customers into investors through innovative service platforms. Journal of Service Management 22(4):443-470.

Pickard, G.; Pan, W.; Rahwan, I.; Cebrian, M.; Crane, R.; Madan, A.; and Pentland, A. 2011. Time-critical social mobilization. Science 334(6055):509-512.

Rzeszotarski, J. M., and Morris, M. R. 2014. Estimating the social costs of friendsourcing. In Proceedings of the 32nd annual ACM conference on Human factors in computing systems, 2735-2744. ACM.

Schmitt, P.; Skiera, B.; and Van den Bulte, C. 2011. Referral programs and customer value. Journal of Marketing 75(1):46-59.

Shang, J., and Croson, R. 2005. Field experiments in charitable contribution: The impact of social influence on the voluntary provision of public goods. Unpublished manuscript.

Shang, J., and Croson, R. 2009. A field experiment in charitable contribution: The impact of social information on the voluntary provision of public goods. The Economic Journal 119(540):1422-1439.

Sleeper, M.; Balebako, R.; Das, S.; McConahy, A. L.; Wiese, J.; and Cranor, L. F. 2013. The post that wasn't: exploring self-censorship on facebook. In Proceedings of the 2013 conference on Computer supported cooperative work, 793802. ACM.

Smith, S.; Windmeijer, F.; and Wright, E. 2014. Peer effects in charitable giving: Evidence from the (running) field. The Economic Journal n/a-n/a. 
In aid of

CANCER RESEARCH

\section{Help fight cancer with just a click}

Southampton

points so far

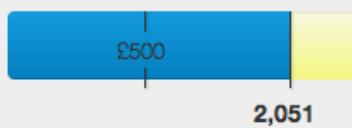

- Did you know that oesophageal cancer is one of the fastest rising and most deadly cancers?

- Six Southampton medics are training for the New York Marathon this November, to raise $£ 100,000$ for oesophageal cancer research.

- We are helping the runners reach their goal by spreading the word.

- You can help by just signing up. It costs you nothing and adds 1 point to the total.

- Once we collect 7,949 more points, we will donate $£ 500$.

- People like you already contributed 2,051 points and we already donated $£ 500$. next target

।

5500

10,000

\begin{tabular}{|c|c|c|}
\hline \multicolumn{2}{|c|}{ Email Address } & (2) \\
\hline \multicolumn{3}{|c|}{ Choose Password } \\
\hline \multicolumn{3}{|c|}{ Confirm Password } \\
\hline \multicolumn{3}{|c|}{ Click to Contribute 1 Point } \\
\hline \multicolumn{3}{|c|}{ Or } \\
\hline f & Support via Facebook & \\
\hline 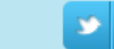 & Support via Twitter & \\
\hline \multicolumn{3}{|c|}{ (Log in for existing users) } \\
\hline
\end{tabular}

\section{Find out more}

By clicking on the buttons below, you can get more information about the research we are supporting, who we are, how this charitable drive works, our data usage policy and our contact details.

What We Suppor
Who We Are
How This Works
Data Usage
Contact Us

Figure 9: The landing page shown to new visitors. 


\section{Thank you very much!}

You have contributed 1 point to fight oesophageal cancer. $£ 500$ will be donated once 7,948 more points are generated.

$$
\text { points so far }
$$

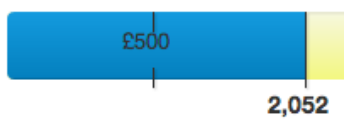

$£ 500$

2,052

\section{Help reach the target faster}

Why not go the extra mile and invite your friends to sign up? This will help the drive reach the highest target and release even more funds. Our next goal is 10,000 points, which will release $£ 500$ for the cause. We've already got the first $£ 500$.

Copy your personal referral link and send it to your friends: (How can I use this?)

$$
\text { http://outruncancer.co.uk/ref/cdbh }
$$

Or use the following buttons:

$$
\text { if Share } c \text { Tweet } c
$$

How much awareness can you generate?

- 0 of your friends signed up

\section{Make a Donation}

You can further support the Cancer Marathon by making a direct donation. If each of us contributes $£ 3$ or $£ 5$, we can make a big difference.

As an additional reward, we will match any donation you make (up to a maximum of $£ 10$ per user). So if you donate $£ 10$, we will also donate $£ 10$, leading to a total donation of £20.

Please enter your donation below and you will be directed to our secure JustGiving page.

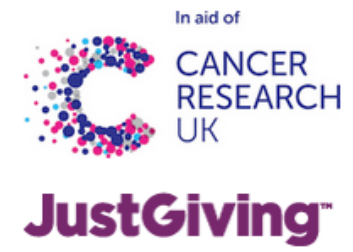

$\begin{aligned} \text { Your Donation: } & £ 10 \\ \text { Our Donation: } & +£ 10 \\ \text { Total Donation: } & =£ 20\end{aligned}$

Donate

Figure 10: Call to action after signing up (control treatment shown). 